\section{Cardiac electrical system involvement in Alström syndrome: uncommon causes of dilated cardiomyopathies}

\author{
Richard J. Czosek, ${ }^{1}$ Paula Goldenberg,' \\ Erin M. Miller, ${ }^{1}$ Robert Spicer, ${ }^{1}$ \\ Jeffrey A. Towbin, ${ }^{1}$ Stephanie M. Ware ${ }^{1,2}$ \\ 'Division of Pediatric Cardiology; \\ 2Division of Molecular Cardiovascular \\ Biology, The Heart Institute, Cincinnati \\ Children's Hospital Medical Center, OH, \\ USA
}

\section{Abstract}

Alström syndrome is a rare autosomal recessive disorder with dilated cardiomyopathy in $60 \%$ of patients. Despite the frequency of cardiac involvement in Alström syndrome, conduction system abnormalities or arrhythmias have not been characterized previously. We report two siblings with Alström syndrome with conduction system involvement with left bundle branch block on electrocardiogram (ECG). One patient had first degree atrioventricular block in addition to bundle branch block and underwent pacemaker implantation. This same patient developed intra-atrial reentry tachycardia requiring anti-arrhythmic medication and eventual trans-catheter ablation. The second patient developed atrial and ventricular arrhythmias and underwent placement of a bi-ventricular defibrillator. These findings suggest that cardiac conduction system involvement and clinical arrhythmia may be significant yet under-recognized complications in patients with Alström syndrome. Patients should be routinely screened with ECG and Holter monitoring in addition to echocardiographic assessment and a cardiologist experienced with cardiomyopathy should be an integral part of the care team.

\section{Introduction}

Alström syndrome is a rare autosomal recessive disorder. Its hallmark features include dilated cardiomyopathy, nystagmus and pigmentary retinopathy, childhood obesity without hyperphagia, and sensorineural hearing loss. This disorder has progressive multi-systemic effects with patients developing metabolic syndrome (hypercholesterolemia, hypertriglyceridemia, insulin resistant diabetes), endocrine (thyroid, growth, sex hormone dys- regulation with hypogonadism), liver, pulmonary, and renal disease over time. Individuals can be identified with developmental delay, which may be due to vision and hearing impairments. It is likely that Alström syndrome is underdiagnosed as individuals with this disorder are frequently followed by various specialty services, without consideration of a unifying diagnosis.

Alström syndrome, an autosomal recessive disorder, is one of a group of disorders of cilia, collectively termed ciliopathies..$^{1-3}$ Mutations of the ALMS1 gene cause Alström syndrome $\mathrm{e}^{4,5}$ The role of this gene in disease pathology is unclear, but it is hypothesized to play a role in intracellular trafficking. ${ }^{6}$ Mouse models (Alms1 -/-) have similar manifestations including obesity, hypogonadism, hyperinsulinemia, retinal dysfunction, and late-onset hearing loss. In these murine models ALMS1 appears to have a role in planar cell polarity signaling in the cochlea, adipogenesis, and rhodopsin trafficking. ${ }^{6-8}$

The diagnosis of Alström syndrome can be difficult because of its rarity and similarity to other syndromic conditions. Bardet-Biedl syndrome is another ciliopathy associated with cone-rod dystrophy, polydactyly, obesity, diabetes, and hypogonadism, however cardiomyopathy is infrequently associated with this condition. Kearns-Sayre syndrome is a progressive mitochondrial disorder associated with pigmentary retinal degeneration, complete heart block, cardiomyopathy, sensorineural hearing loss, diabetes mellitus, and renal Fanconi disease that can have a very similar phenotypic presentation.

Despite the frequent incidence of dilated cardiomyopathy reported in patients with Alström syndrome (60\%), ${ }^{6}$ conduction system involvement or cardiac arrhythmias have not been described in this patient population to date and the longitudinal cardiac features have been poorly documented. We report two siblings with Alström syndrome whose phenotype included conduction system involvement and significant clinical arrhythmias.

\section{Case Reports}

\section{Case \#1}

The first patient is a 20 -year-old male who initially presented to cardiology at the age of 17 years for tachycardia (Figure 1A). Prior to his evaluation by cardiology, he had been diagnosed with retinitis pigmentosa and had severe visual impairment. Upon presentation he had moderate ventricular dysfunction with a shortening fraction (SF) of $23 \%$ and mild left atrial enlargement (Figure 2A). During this admission, the patient was started on digoxin for treatment of his tachycardia. At his initial
Correspondence: Richard J. Czosek, Cincinnati Children's Hospital Medical Center, Pediatric Cardiology, MLC 2003, 3333 Burnett Avenue, Cincinnati, OH 45229, USA.

Tel. +1.513.636.2237 - Fax: +1.513.636.7996.

E-mail: richard.czosek@cchmc.org

Funding: JAT and SMW are funded by National Institutes of Health grants R01 HL087000-01A1 (JAT, SWM), the Children's Cardiomyopathy Foundation (SMW), and Burroughs Wellcome Fund Clinical Scientist Award in Translational Research \#1008496 (SMW).

Key words: Alström syndrome, arrhythmia, cardiomyopathy.

Contributions: RJC, PG, EM, RS, JAT, SMW, data acquisition, analysis and interpretation, manuscript drafting/revision; RJC, SMW, project conception and design. All authors have approved the final version of the manuscript.

Conflicts of interests: the authors declare no potential conflicts of interests.

Received for publication: 7 November 2011

Revision received: 16 January 2012.

Accepted for publication: 17 January 2012.

This work is licensed under a Creative Commons Attribution NonCommercial 3.0 License (CC BYNC 3.0).

(C) Copyright R.J. Czosek et al., 2012

Licensee PAGEPress, Italy

Cardiogenetics 2012; 2:e2

doi:10.4081/cardiogenetics.2012.e2

outpatient follow-up visit, he demonstrated improved ventricular function with a shortening fraction of $30 \%$. He was initially diagnosed with tachycardia-mediated cardiomyopathy. His cardiac function was thought to likely improve in time with management of the arrhythmia.

Six months later he was noted to have concerning left ventricular (LV) dilation and a progressive widening of his QRS interval (148 ms combined; PR interval of $196 \mathrm{~ms}$ ) with stable function (Figure 3A). These new electrocardiogram (ECG) findings in the context of his previously noted retinitis pigmentosa raised concern for Kearns-Sayre syndrome. He was referred to a pediatric geneticist and a cardiologist with cardiomyopathy experience. On exam he was noted to have short stature (2\%ile). Additional testing demonstrated high frequency sensorineural hearing loss and glucose intolerance. mtDNA testing in blood for common deletions/duplications including Kearns-Sayre syndrome was normal. A cardiac biopsy showed displacement of myofibrils. By electron microscopy, the intermyofibril spaces were filled with increased numbers of mitochondria with striking proliferative features and, in some 
areas, glycogen granules, consistent with a mitochondrial disorder. He developed progressive first degree AV block, and underwent placement of a dual chamber pacemaker. A skeletal muscle biopsy was performed while under anesthesia for his pacemaker placement. Testing of the muscle biopsy for deletions, the gold standard diagnostic test for Kearns-Sayre, was normal. Given this patient's constellation of symptoms, Alström syndrome was considered. He was found to have two ALMS1 mutations (c.7369_7370delGA (p.Asp2457Stop) and c.7374_7375delGA (p.Asp2459Stop)) which confirmed a diagnosis of Alström syndrome.

Over the next two years this patient developed progressive QRS widening with a left bundle branch block pattern and was admitted for atrial flutter which was successfully cardioverted. Five months following his device implantation he developed atrial flutter refractory to electrical cardioversion or amiodarone therapy, and required trans-venous ablation of several intra-atrial reentry circuits (Figure 3B). To date he remains clinically stable in sinus rhythm with mild LV dysfunction on a medical regimen of atenolol, lisinopril, metformin and lasix.

\section{Case \#2}

The second patient is a 25-year-old female (Figure 1B) who initially presented for cardiac evaluation at the age of 23 years following the diagnosis of dilated cardiomyopathy in her younger brother (Patient \#1). Prior to evaluation in the cardiology clinic, she had been diagnosed with retinitis pigmentosa, systemic hypertension and Type II diabetes mellitus. Upon initial evaluation, her echocardiogram demonstrated borderline normal ventricular function with a SF of $27 \%$ and mild left atrial dilation (Figure 2B). Initial ECG demonstrated a left bundle branch block pattern with a QRS duration of $125 \mathrm{~ms}$ (Figure 3C). She was started on carvedilol in addition to her prior regimen of lisinopril and metformin.

ALMS1 testing was performed on this patient for her known familial mutations which confirmed a diagnosis of Alström syndrome. Since her diagnosis she has developed progressive LV dysfunction, with a current SF of $19 \%$ and ejection fraction $<35 \%$. Associated with the decline in cardiac function, she developed frequent episodes of non-sustained ventricular tachycardia. Secondary to the combination of poor systolic function, left bundle branch block and ventricular arrhythmias she underwent placement of a bi-ventricular defibrillator system. Subsequently, she went on to develop recurrent atrial flutter and is currently well controlled on Amiodarone.

\section{Materials and Methods}

Patient, family and medical history were evaluated in two siblings with Alström syndrome including genetic, echocardiographic and electrophysiological data. Patient information was collected as part of a clinical registry study approved by the Institutional Review Board of Cincinnati Children's Hospital. Patient consent was obtained for photography and publication.

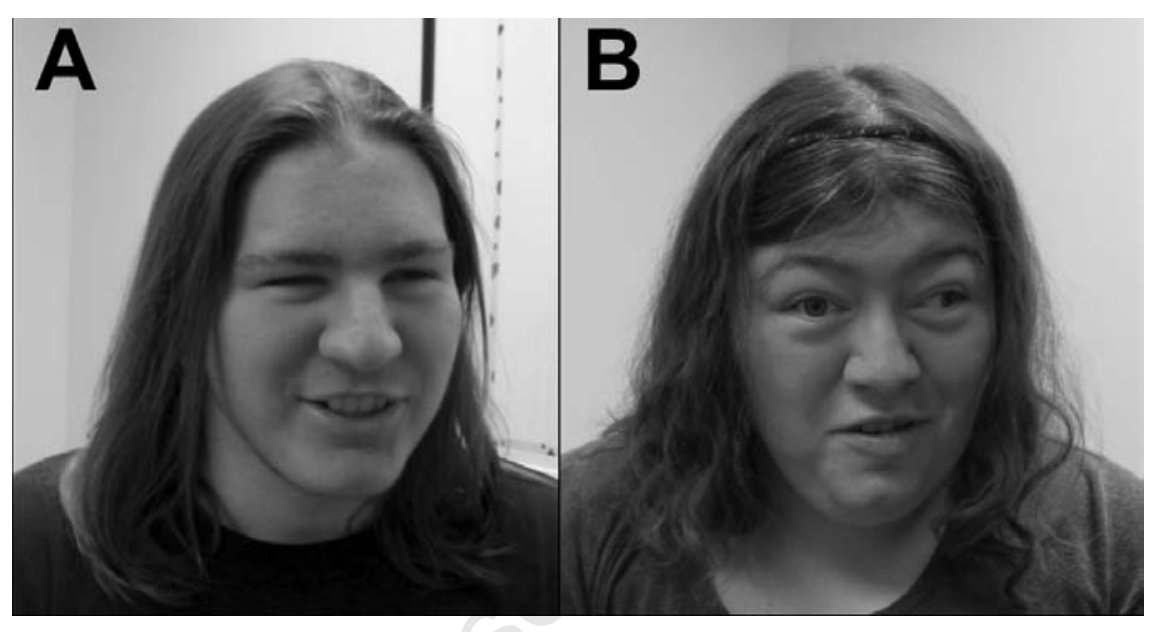

Figure 1. Clinical facial features of described patients with Alström syndrome. A) Case \#1; B) Case \#2.
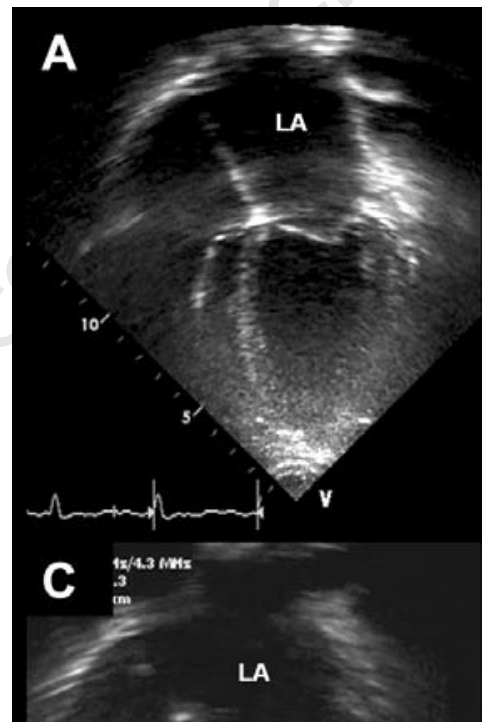

LA

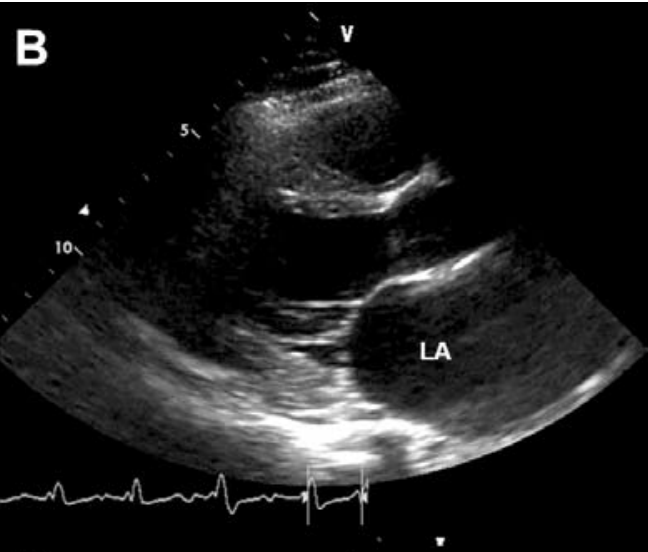

D
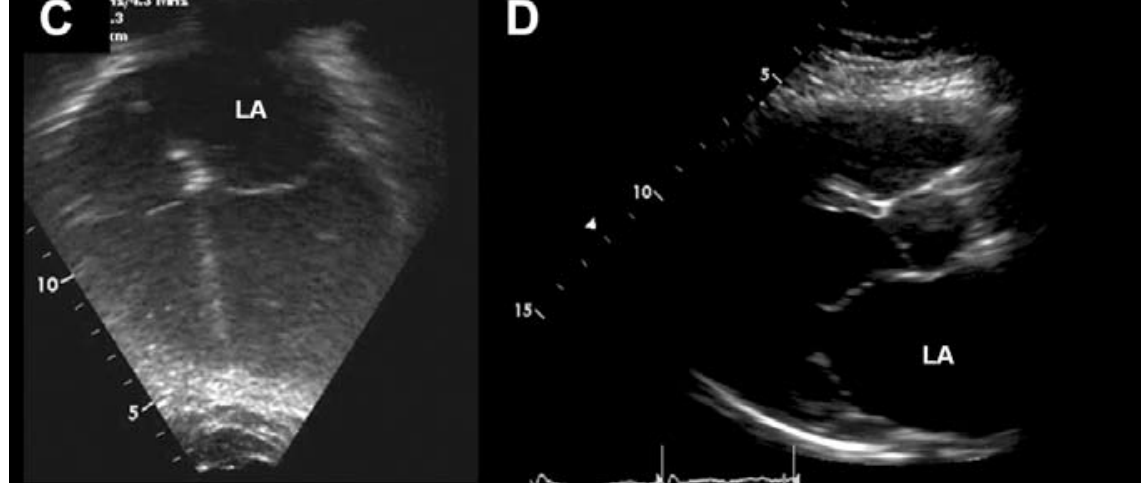

Figure 2. Patient phenotype and echocardiogram. A) Four chamber view of Case \#1. Note the dilated cardiac phenotype as well as the dilated left atrium; B) Long axis view of Case \#1. C) Four chamber view of Case \#2. Note the dilated cardiac phenotype as well as the dilated left atrium; D) Long axis view of Case \#2. LA, left atrium. 


\section{Discussion}

Dilated cardiomyopathy is a frequently heritable and familial condition, however a pathogenic mutation is found in only approximately $30-40 \%$ of patients tested. ${ }^{9}$ In the pediatric age group there are both genetic and environmental causes of dilated cardiomyopathy. Genetic causes include mitochondrial disorders, fatty acid oxidation defects, genetic syndromic conditions, or mutations in sarcomeric or cytoskeletal genes. Although most genetic syndromes associated with dilated cardiomyopathy are rare, in aggregate they account for a substantial fraction of disease. Ciliopathies are an increasingly recognized group of heterogenous genetic disorders. The molecular mechanism underlying dilated cardiomyopathy in Alström syndrome is not known. Furthermore, there is a paucity of information on cardiac involvement in other ciliopathies. Cardiomyopathy has been described in a subset of patients with Leber's congenital amaurosis. ${ }^{10}$ A small case series described 2/8 patients with Bardet-Biedl syndrome with cardiomyopathy but provided no additional details. ${ }^{3}$ Finally, Elbedour et al. documented cardiac involvement in 50\% of Bardet-Biedl patients including congenital heart defects, hypertrophy of the interventricular septum, and dilated cardiomyopathy, and recommended ongoing cardiac surveillance. ${ }^{11}$ Given that many of the ciliopathies share clinical and etiologic features, the possibility of cardiac dysfunction in other ciliopathies needs further evaluation.

Cardiac conduction system disease is often associated with various forms of cardiomyopathy, and clinical arrhythmias are frequently degenerative. ${ }^{12}$ In specific subtypes of cardiomyopathies electrocardiographic abnormalities may precede echocardiogram evidence of functional abnormality. ${ }^{13}$ These ECG findings have been associated with worse patient outcome. ${ }^{14-17}$ Thus, an abnormal ECG may be a prognostic indicator for development of future ventricular dysfunction and identify a high risk group of patients within the larger population of patients with cardiomyopathy. The mechanisms underlying conduction system abnormalities in patients with Alström syndrome are not known and potentially multi-factorial. Similar to central conduction system disease, arrhythmias may occur either as a result of the underlying muscle cell abnormality or as a result of poor systolic function and ventricular dilation in patients with cardiomyopathy. ${ }^{18}$ Although other ciliopathies syndromes including Bardet-Biedl and Kartagener's syndrome have been associated with significant cardiac involvement, potential conduction system and arrhythmia involvement remains unknown. ${ }^{19}$ Accurate description of the frequency of ECG abnormalities and associated arrhythmias in patients with Alström syndrome compared to other cardiomyopathy cohorts is needed to delineate whether these abnormalities are secondary to the dilated cardiomyopathy phenotype or potentially related to the ALMS1 mutation itself.

Previously it was thought that ventricular dysfunction diagnosed during infancy in Alström syndrome was transient. ${ }^{20}$ More recent reports have shown that in up to $30 \%$ of patients normalization of ventricular function is merely a quiescent period, with cardiomyopathy recurring in adolescence or adulthood. ${ }^{6}$ The presence of cardiomyopathy in adolescent
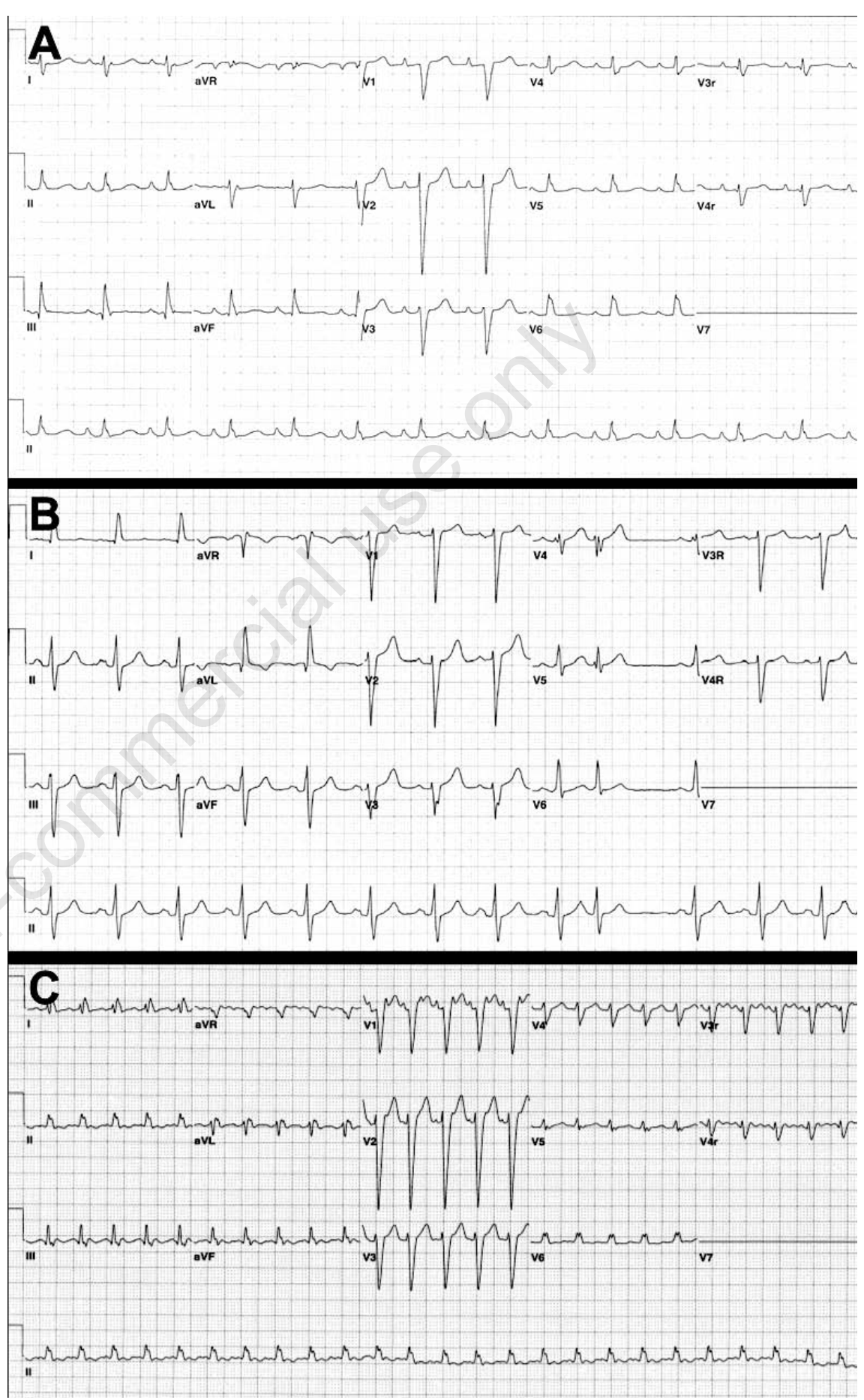

Figure 3. Surface electrocardiograms. A) Case \#1. Left bundle branch block pattern with a QRS duration of $142 \mathrm{~ms}$ and a PR interval of $200 \mathrm{~ms}$; B) Case \#2. Left bundle branch block pattern with a QRS duration of $125 \mathrm{~ms}$; C) Intra-atrial reentry tachycardia in Case \#1. Atrial rate of 300 beats per minute with 2:1 atrioventricular conduction and a ventricular response rate of 150 beats per minute 
and adult patients with Alström syndrome is associated with a poor prognosis and significant mortality. In response to the significant incidence of cardiomyopathy in patients with Alström syndrome, serial echocardiographic assessment has been recommended as part of routine patient care. ${ }^{21}$ Although cardiac involvement is a common finding in patients with Alström syndrome, there has been no previous description of the central conduction system or cardiac rhythm disturbances in this patient population. This report represents the first description of both central conduction system disease and cardiac rhythm abnormalities in patients with Alström syndrome. Whether conduction system abnormalities are truly rare or whether there is under-reporting or underrecognition of these defects is unknown and more detailed analysis of ECG and arrhythmic involvement in larger Alström populations is needed.

Early detection of conduction defects in patients with Alström syndrome would allow for earlier and more aggressive treatment reducing morbidity and mortality. Prior studies in a variety of cardiomyopathy subgroups have demonstrated that preceding and predictive ECG changes can be used as a prognostic tool indicating the need for early treatment of cardiomyopathy. ${ }^{22}$ Hombach et al. reported that a QRS interval $>110 \mathrm{~ms}$ is an independent predictor of both overall cardiac death as well as sudden cardiac death in a large cohort of adult cardiomyopathy patients. Whether ECG abnormalities precede echocardiographic evidence of ventricular dysfunction in a substantial number of patients with Alström syndrome will need to be evaluated.

In addition to the increased risk of cardiac death, significant conduction system delay also results in ventricular dyssynchrony and affects ventricular-ventricular interaction. Ventricular resynchronization has been found to improve functional outcomes and survival in adults with cardiomyopathy, ${ }^{23-27}$ and biventricular pacing has improved cardiac function in pediatric patients with cardiomyopathy. ${ }^{28-30}$ Given that both patients described in this report had early documentation of left bundle branch block, larger cohorts of patients with Alström syndrome should be evaluated with regard to conduction system involvement and possible response rates to cardiac resynchronization therapy. It has been our practice to follow published indications for both ICD and/or biventricular resynchronization therapy based on cardiac dysfunction and QRS duration. ${ }^{31-33}$

Delay in diagnosis can delay early intervention and management of patients with ciliopathies such as Alström syndrome. Several of the ciliopathies that are associated with dilated cardiomyopathy have overlapping extra-cardiac findings with Kearns-Sayre syn- drome. Although there is already a significant burden of medical care on these patients due to the multiple subspecialties involved, early detection of subtle cardiac findings may significantly impact patient management and eventual outcomes. Because of this potential impact, we recommend the involvement of a cardiologist experienced in treating patients with cardiomyopathies and their associated complications.

\section{Conclusions}

Patients with Alström syndrome are at risk of developing abnormalities of the central conduction system and clinical arrhythmias in addition to dilated cardiomyopathy. As with all forms of cardiomyopathy, we recommend routine ECG and ambulatory monitoring in all patients with Alström syndrome. In addition, a cardiologist with expertise in patients with cardiomyopathies should be an integral part of the treatment team in patients with Alström syndrome.

\section{References}

1. Cardenas-Rodriguez M, Badano JL. Ciliary biology: understanding the cellular and genetic basis of human ciliopathies. Am J Med Genet C Semin Med Genet 2009; 151C:263-80.

2. Baker K, Beales PL. Making sense of cilia in disease: the human ciliopathies. Am J Med Genet C Semin Med Genet 2009;151C: 281-95.

3. Beales PL, Elcioglu N, Woolf AS, et al. New criteria for improved diagnosis of BardetBiedl syndrome: results of a population survey. J Med Genet 1999;36:437-46.

4. Marshall JD, Hinman EG, Collin GB, et al. Spectrum of ALMS1 variants and evaluation of genotype-phenotype correlations in Alstrom syndrome. Hum Mutat 2007;28: 1114-23.

5. Collin GB, Marshall JD, Ikeda A, et al. Mutations in ALMS1 cause obesity, type 2 diabetes and neurosensory degeneration in Alstrom syndrome. Nat Genet 2002;31: 74-8.

6. Marshall JD, Bronson RT, Collin GB, et al. New Alstrom syndrome phenotypes based on the evaluation of 182 cases. Arch Intern Med 2005;165:675-83.

7. Huang-Doran I, Semple RK. Knockdown of the Alstrom syndrome-associated gene Alms1 in 3T3-L1 preadipocytes impairs adipogenesis but has no effect on cellautonomous insulin action. Int $\mathrm{J}$ Obes (Lond) 2010;34:1554-8.
8. Jagger D, Collin G, Kelly J, et al. Alstrom Syndrome protein ALMS1 localizes to basal bodies of cochlear hair cells and regulates cilium-dependent planar cell polarity. Hum Mol Genet 2011;20:466-81.

9. Towbin JA, Lowe AM, Colan SD, et al. Incidence, causes, and outcomes of dilated cardiomyopathy in children. JAMA 2006;296:1867-76.

10. Russell-Eggitt IM, Taylor DS, Clayton PT, et al. Leber's congenital amaurosis--a new syndrome with a cardiomyopathy. $\mathrm{Br} \mathrm{J}$ Ophthalmol 1989;73:250-4.

11. Elbedour K, Zucker N, Zalzstein E, et al. Cardiac abnormalities in the Bardet-Biedl syndrome: echocardiographic studies of 22 patients. Am J Med Genet 1994;52:164-9.

12. Okutucu S, Oto A. Risk stratification in nonischemic dilated cardiomyopathy: Current perspectives. Cardiol J 2010;17: 219-29.

13. Shah AM, Jefferies JL, Rossano JW, et al. Electrocardiographic abnormalities and arrhythmias are strongly associated with the development of cardiomyopathy in muscular dystrophy. Heart Rhythm 2010;7: 1484-8.

14. Groh WJ, Groh MR, Saha C, et al. Electrocardiographic abnormalities and sudden death in myotonic dystrophy type 1. N Engl J Med 2008;358:2688-97.

15. Mestroni L, Neri R, Camerini F. [The electrocardiogram in dilated cardiomyopathy]. G Ital Cardiol 1986;16:1009-17. [Article in Italian ]

16. Huh J, Noh CI, Yun YS. The usefulness of surface electrocardiogram as a prognostic predictor in children with idiopathic dilated cardiomyopathy. J Korean Med Sci 2004;19:652-5.

17. Cianfrocca C, Pelliccia F, Nigri A, Critelli G. Resting and ambulatory ECG predictors of mode of death in dilated cardiomyopathy. $\mathrm{J}$ Electrocardiol 1992;25:295-303.

18. Friedman RA, Moak JP, Garson A Jr. Clinical course of idiopathic dilated cardiomyopathy in children. J Am Coll Cardiol 1991;18:152-6.

19. Deveault C, Billingsley G, Duncan JL, et al. BBS genotype-phenotype assessment of a multiethnic patient cohort calls for a revision of the disease definition. Hum Mutat 2011;32:610-9.

20. Michaud JL, Heon E, Guilbert F, et al. Natural history of Alstrom syndrome in early childhood: onset with dilated cardiomyopathy. J Pediatr 1996;128:225-9.

21. Marshall JD, Beck S, Maffei P, Naggert JK. Alstrom syndrome. Eur J Hum Genet 2007; 15:1193-202.

22. Hombach V, Merkle N, Torzewski J, et al. Electrocardiographic and cardiac magnetic resonance imaging parameters as predictors of a worse outcome in patients with 
idiopathic dilated cardiomyopathy. Eur Heart J 2009;30:2011-8.

23. Stellbrink C, Breithardt OA, Franke A, et al. Impact of cardiac resynchronization therapy using hemodynamically optimized pacing on left ventricular remodeling in patients with congestive heart failure and ventricular conduction disturbances. J Am Coll Cardiol 2001;38:1957-65.

24. Manolis AS. Cardiac resynchronization therapy in congestive heart failure: Ready for prime time? Heart Rhythm 2004;1:35563.

25. Gasparini M, Auricchio A, Regoli F, et al. Four-year efficacy of cardiac resynchronization therapy on exercise tolerance and disease progression: the importance of performing atrioventricular junction ablation in patients with atrial fibrillation. $\mathrm{J}$ Am Coll Cardiol 2006;48:734-43.

26. Molhoek SG, Bax JJ, van Erven L, et al.
Comparison of benefits from cardiac resynchronization therapy in patients with ischemic cardiomyopathy versus idiopathic dilated cardiomyopathy. Am J Cardiol 2004;93:860-3.

27. Molhoek SG, Bax JJ, Bleeker GB, et al. Long-term follow-up of cardiac resynchronization therapy in patients with endstage heart failure. J Cardiovasc Electrophysiol 2005;16:701-7.

28. Greene EA, Berul CI. Pacing treatment for dilated cardiomyopathy: optimization of resynchronization pacing in pediatrics. Curr Opin Cardiol 2010;25:95-101.

29. Cecchin F, Frangini PA, Brown DW, et al. Cardiac resynchronization therapy (and multisite pacing) in pediatrics and congenital heart disease: five years experience in a single institution. $\mathrm{J}$ Cardiovasc Electrophysiol 2009;20:58-65.

30. Janousek J, Gebauer RA, Abdul-Khaliq H, et al. Cardiac resynchronisation therapy in paediatric and congenital heart disease: differential effects in various anatomical and functional substrates. Heart 2009;95:1165-71.

31. Goldberger Z, Lampert R. Implantable cardioverter-defibrillators: expanding indications and technologies. JAMA 2006;295: 809-18.

32. Tang AS, Wells GA, Talajic M, et al. Cardiac-resynchronization therapy for mild-to-moderate heart failure. $\mathrm{N}$ Engl $\mathrm{J}$ Med 2010;363:2385-95.

33. Linde C, Abraham WT, Gold MR, et al. Randomized trial of cardiac resynchronization in mildly symptomatic heart failure patients and in asymptomatic patients with left ventricular dysfunction and previous heart failure symptoms. J Am Coll Cardiol 2008;52:1834-43. 